www.jmscr.igmpublication.org

Impact Factor 5.84

Index Copernicus Value: 71.58

ISSN (e)-2347-176x ISSN (p) 2455-0450

crossref DOI: _https://dx.doi.org/10.18535/jmscr/v5i10.139

Journal Of Medical Science And Clinical Research

\title{
Myocardial Protection in Aortic Valve Replacement with Severe Aortic Stenosis: A Comperative Study between Del Nido and HTK Solution
}

Authors

\section{Kamal Das ${ }^{1}$, Pritam Nandy ${ }^{2}$, Tuhinsubhra Medda ${ }^{3}$, Nripendra kr. Tiwari ${ }^{4}$, Suipta Patra ${ }^{5}$, Dr Bhaskar Das ${ }^{6}$, Dr Rajarshi Basu ${ }^{7}$}

${ }^{1}$ MSc in Perfusion Sciences, Chief Perfusionist, Department of Cardiothoracic \& Vascular Surgery, R G Kar Medical College, Kolkata

${ }^{2,3,4} 3$ rd yr BSc in Perfusion Technology, Department of Cardiothoracic \& Vascular Surgery, R G Kar

Medical College, Kolkata

${ }^{5} 1$ st yr MSc in Perfusion Science, Department of Cardiothoracic \& Vascular Surgery, R G Kar Medical College, Kolkata

${ }^{6}$ Assistant Professor, Department of Cardiothoracic \& Vascular Surgery, R G Kar Medical College, Kolkata ${ }^{7}$ Associate Professor Department of Cardiothoracic \& Vascular Surgery, R G Kar Medical College, Kolkata

\begin{abstract}
Both Delnido and HTK Cardioplegia provides excellent myocardial protection and reduces the frequency of cardioplegia administration which allows uninterrupted operation and also reduces ' $x$ '-clamp timing. Both helps smooth myocardial recovery with post operative minimal inotropic support. But in aortic valve replacement in severe aortic stenosis with hypertrophied myocardium delnido cardioplegia provides poor protection where HTK provides better.

Material and Methods: Twenty adult patients devided into two groups, Group-A $(n=10)$ received delnido cardioplegia and Group-B $(n=10)$ received HTK cardioplegia, underwent aortic valve replacement in severe aortic stenosis with hypertrophid myocardium. In both group same dose of cardioplegia (20ml/kg) was administered through anterograde route.

Results: In group $-B$ after valve replacement cardiac function was satisfactory with good ejection fraction, no rhythm disturbances occurred, and hemodynamics was well maintained with minimum inotropic support where in group-A in 40\%cases VT/VF, in 20\% case low cardiac output, $60 \%$ cases needed high inotrope with prolonged ventilatory support.

Conclusion: HTK cardioplegia provides better myocardial protection in aortic valve replacement in severe aortic stenosis with hypertrophied myocardium.

Keywords: myocardial protection, del nido cardioplegia, htk cardioplegia, aortic valve surgery.
\end{abstract}

\section{Introduction}

Various Cardioplegic solutions are used to arrest the heart and to preserve the myocardium. Commonly the St. Thomas potassium containing extracellular cardioplegia is used in most of the cases. The use of Delnido cardioplegia also increased now a days. The intracellular cardioplegia, HTK mainly used for long time myocardial protection and organ preservation. It is very essential to protect the myocardium because the 
cardiac function and post operative outcome depends on it.

Cardiopulmonary bypass, ' $X$ '-clamping the aorta, reperfusion injury and myocardial oedema are the insulting factors to the myocardial function insufficiency.

Moreover, the hypertrophic heart has different metabolic demands than other pathologies and, therefore, may have different susceptibility to ischaemia and reperfusion.

In progression of myocardial protection include one or more combinations of warm versus cold blood cardioplegia, antegrade versus retrograde delivery, intermittent versus continuous perfusion reducing $\mathrm{Ca} 2+$ overload, provide energy substrates and remove harmful metabolic substances.

The del Nido cardioplegia solution has been in use for 18 years at Boston Childre's Hospital. Although it was originally developed for paediatric and infant patients, it's use for adult cardiac surgery has been expanding. Del Nido cardioplegia solution provides a depolarized hyperkalemic arrest lasting up to 60 minutes ${ }^{(1)}$, and the addition of lidocaine and Magnesium Sulphate may limit intracellular calcium influx ${ }^{(2)}$. Single-dose delnido cardioplegia solution may offer an alternative myocardial protection strategy to multi-dose cold whole blood (WB) cardioplegia (3-5).

HTK cardioplegia also attractive as a single dose provides a long period of myocardial protection, it adopted widely not only in cardiac transplantation but also in the preservation of multiple $\operatorname{organs}^{(6)}$. Histidine-triptophan-ketoglutarate (HTK) solution based on intracellular level of electrolytes, proposed by Bretschneider in the year $1970^{(7)}$. Histidine, tryptophan, ketoglutarate, and mannitol act as buffers, improve high energy production by ATP during reperfusion, stabilize cell membranes, and maintain osmotic regulation of the cell membrane ${ }^{(7)}$. For these reason being these two types of cardioplegia have been used in our study.
Table 1-Components of Del Nido Cardioplegia

\begin{tabular}{|ll|}
\hline Plasmalyte A & $1000 \mathrm{ml}$ \\
Mannitol (20\%) & $16.3 \mathrm{ml}$ \\
Magnesium Sulfate $(50 \%)$ & $4 \mathrm{ml}$ \\
Sodium Bicarbonate $(8.4 \%)$ & $13 \mathrm{ml}$ \\
Potassium Chloride $(2 \mathrm{meq} / \mathrm{ml})$ & $13 \mathrm{ml}$ \\
Lidocaine (2\%) & $6 \mathrm{ml}$ \\
\hline
\end{tabular}

Base solution Plasmalyte-A- The del Nido cardioplegia contains a base solution PlasmalyteA, which contains $140 \mathrm{mEq} / \mathrm{L}$ sodium, $5 \mathrm{mEq} / \mathrm{L}$ potassium, $3 \mathrm{mEq} / \mathrm{L}$ magnesium, $98 \mathrm{mEq} / \mathrm{L}$ chloride, $27 \mathrm{mEq} / \mathrm{L}$ acetate, $23 \mathrm{mEq} / \mathrm{L}$ gluconate and $\mathrm{pH} 7.4$.

Table 2- Components of HTK cardioplegia.

\begin{tabular}{|ll|}
\hline Components & Value \\
Histidine & $198 \mathrm{mmol} / \mathrm{L}$ \\
Tryptophan & $2 \mathrm{mmol} / \mathrm{L}$ \\
Ketoglutarate & $1 \mathrm{mmol} / \mathrm{L}$ \\
$\mathrm{Na}+$ & $15 \mathrm{mmol} / \mathrm{L}$ \\
$\mathrm{K}+$ & $9 \mathrm{mmol} / \mathrm{L}$ \\
$\mathrm{Mg} 2+$ & $4 \mathrm{mmol} / \mathrm{L}$ \\
$\mathrm{Ca} 2+$ & $0.015 \mathrm{mmol} / \mathrm{L}$ \\
$\mathrm{Mannitol}$ & $30 \mathrm{mmol} / \mathrm{L}$ \\
$\mathrm{pH}$ & $7.02-7.20$ \\
\hline
\end{tabular}

\section{Material and Methods}

This study was conducted at R.G.Kar Medical College, Kolkata, between January, 2014 and June, 2017.Twenty consecutive patients of aortic valve disease (aortic stenosis) were included in this study. We have used the statistical analysis by software 'R'2.15.2 version.

\section{Pre operative cardiac assesment}

TTE (Trans thoracic echo cardigraphy) done and measured the chamber diameter, orifice size, Ejection Fraction(EF).

\section{Intra operative cardiac assesment}

TEE (Trans Esophageal Echo Cardiography) was done at pre-induction, post induction, after removing of ' $\mathrm{X}$ '-clamp, before and after weaning from CPB (Cardiopulmonary Bypass) and measured the chamber diameter, Ejection Fraction (EF), Cardiac output. . 
All these patients of both group having severe aortic stenosis and eight cases were associated with concentric left ventricular hypertrophy (3 cases of Gr-A and 5 cases of Gr-B).

All surgeries of both groups were performed using a standard general anesthesia protocol, median sternotomy approach, employing cardiopulmonary bypass with moderate systemic hypothermia at 28 to $32^{\circ} \mathrm{C}$. Intraoperative transesophageal echocardiography was routinely employed. Myocardial protection was achieved with delnido/ HTK cardioplegia $20 \mathrm{ml} / \mathrm{Kg}$ body weight (14) over 3 to 5 minutes at the rate of $200-250 \mathrm{ml} / \mathrm{min}$ through antegrade route. Topical hypothermia was performed by cold ice saline intermittently. Pump primed with RL/RI, Mannitol, NaHco3, Heparin. Membrane oxygenator, arterial filter, customised tubings, aortic arch cannula, dual stage venous drainage canula, antegrade cardioplegia canula and non pulsatile pump flow were used in both group. Pump Flow was maintained at 1.6-2.4 lit/minute/m2 BSA and MAP 50-70mmHg was maintained. Pump flow and gas flow ratio was 1:1 to $1: 0.8$, Fio2 $40-60 \%$,PO2 $250-350 \mathrm{mmHg}$ were maintained.

In Group-A $\quad(n=10), \quad$ anterograde delnido cardioplegia with plasmalyte-A was delivered at $20 \mathrm{ml} / \mathrm{Kg}$ body weight at $4^{\circ} \mathrm{c}$ temperature, loaded in $50 \mathrm{ml}$ syringe and was connected with $1 / 4$ inch luer lock connector after the cardioplegia delivery pump. The plasmalyte solution bag was connected pre plegia pump line with infusion set and 3-way stopcock,. Delnido solution was mixed up manually and flow rate $200-250 \mathrm{ml} /$ minute, with pressure 50-70 $\mathrm{mmHg}$ through anterograde route, very simple method (Fig-1).
Fig: 1 Schematic diagram of Delnido Cardioplegia delivery system.

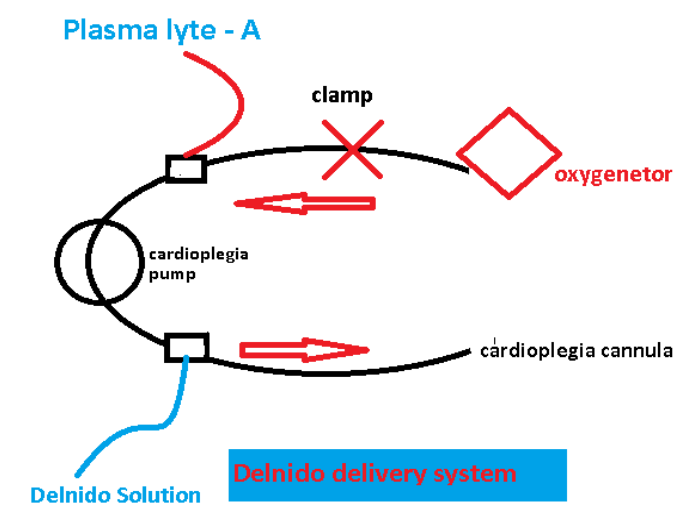

In Group-B ( $\mathrm{n}=10)$, HTK solution bag connected to the pre plegia pump and delivered at $200 \mathrm{ml} / \mathrm{minute}, 20 \mathrm{ml} / \mathrm{kg}$ body weight or maximum $1 \mathrm{~L}$ at $4^{\circ} \mathrm{c}$ temperature. Through plegia pump to the anterograde route (Fig-2).

Fig:2 Schematic diagram of HTK delivery system.

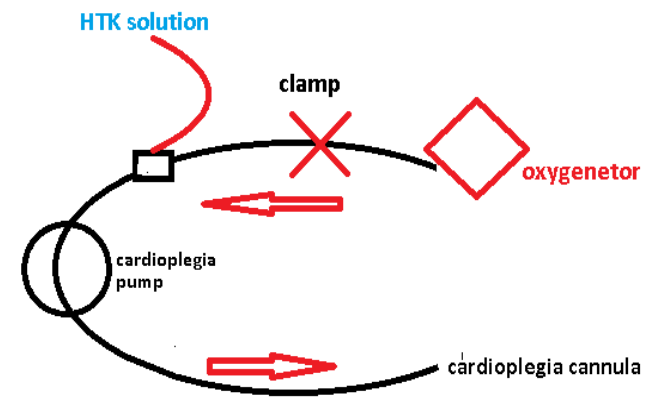

Before declamping of aorta venting was started and continued until coming off the CPB. Ventilation was started, patient is on trendelenberg position then removed the cross clamp. All parameters and investigations were checked and patient was haemodynamically stable and resting time was over then weaning procedure was started. Inotropes, vasodilators were used and gradually wean the patient from CPB. In VT/VF defibrillation and anti arrhythmic drugs (lignocaine, amiodarone) were applied. For difficult weaning, inotropic support was maximised. 


\section{Result}

Out of 20 patients undergoing aortic valve replacement, $14(70 \%)$ were male and remaining 6 $(30 \%)$ were female. All of them were 28 years to 50 years of age.

In Gr-A ( $\mathrm{n}=10), 4(40 \%)(\mathrm{p}<0.05)$ cases presented with VT/VF after removal of ' $\mathrm{X}$ '-clamp and were managed by DC shock (20-40jule biphasic DC shock) followed by antiarrhythmic drugs lignocaine, amiodarone bolous or bolus and followed by infusion. 2(20\%) cases with low cardiac output and managed by high inotropic support. Post operative ventilatory support was longer ( $\mathrm{p}<0.05) .2(20 \%)$ patients died in 1 st and 2nd post operative day due to cardiac failure. Both of these two patients have concentric left ventricular hypertrophy.

In Gr-B there was no evidence of cardiac function abnormalities and mortality. All 10 patients were recovered very smoothly. Majority of them, in $80 \%$ cases with minimum inotropic support (Dopamin $=2-5 \quad \mathrm{micg} / \mathrm{Kg} / \mathrm{min}$, Adrenaline $=0.05 \pm \mathrm{micg} / \mathrm{Kg} / \mathrm{min}$ ) where as in $20 \%$ cases required moderate inotropic support (Dopamine $=5 \pm 2 \mathrm{micg} / \mathrm{Kg} / \mathrm{min}$, Adrenaline $=0.01 \pm 0.05 \mathrm{micg} / \mathrm{Kg} / \mathrm{min})$.Ventilatory support was also lesser $(\mathrm{p}<0.05)$

Table-1 Comparison between HTK and Delnido solution.

\begin{tabular}{|c|c|c|c|c|c|c|c|c|c|c|c|}
\hline \multirow[t]{2}{*}{$\begin{array}{l}\text { Type of } \\
\text { patient }\end{array}$} & \multirow[t]{2}{*}{$\begin{array}{l}\text { No of } \\
\text { cases }\end{array}$} & \multirow[t]{2}{*}{$\begin{array}{c}\text { Cross- } \\
\text { Clamp time }\end{array}$} & \multirow{2}{*}{$\begin{array}{l}\text { Pre-op } \\
\text { cardiac } \\
\text { function }\end{array}$} & \multicolumn{2}{|c|}{$\begin{array}{l}\text { Cardiac function during weaning } \\
\text { from CPB }\end{array}$} & \multicolumn{2}{|c|}{ Inotropic support } & \multicolumn{2}{|c|}{ Ventilatory support } & \multicolumn{2}{|c|}{ Mortality } \\
\hline & & & & Gr-A(n=10) & $\begin{array}{c}\text { Gr-B } \\
(\mathrm{n}=10)\end{array}$ & $\begin{array}{c}\text { Gr- } \\
\mathrm{A}(\mathrm{n}=10)\end{array}$ & Gr-B $(n=10)$ & $\begin{array}{c}\text { Gr- } \\
\mathrm{A}(\mathrm{n}=10)\end{array}$ & $\begin{array}{c}\text { Gr-B } \\
(\mathrm{n}=10)\end{array}$ & $\begin{array}{c}\mathrm{Gr}- \\
\mathrm{A}(\mathrm{n}=10)\end{array}$ & $\begin{array}{c}\mathrm{Gr}-\mathrm{B} \\
(\mathrm{n}=10)\end{array}$ \\
\hline $\begin{array}{l}\text { Aortic } \\
\text { Stenosis }\end{array}$ & 20 & $\begin{array}{c}58 \pm 10 \\
\text { minutes in } \\
\text { both group }\end{array}$ & $\begin{array}{c}\mathrm{EF} \\
>50 \%\end{array}$ & $\begin{array}{c}\mathrm{VT} / \mathrm{VF}=4(40 \%) \\
\mathrm{EF} \& \mathrm{CO} \text { low=in } \\
2(20 \%) \text { cases, } \\
\text { Normal=4(40\%) } \\
\text { cases }\end{array}$ & $\begin{array}{c}\mathrm{VT} / \mathrm{VF}=\text { nil } \\
\mathrm{CO}=\text { norma } \\
1 \\
\mathrm{EF}=\text { normal }\end{array}$ & $\begin{array}{c}\text { High }=6(6 \\
0 \%) \\
\text { Mod=4(4 } \\
0 \%)\end{array}$ & $\begin{array}{l}\text { Mod }=2(20 \%) \\
\text { Low }=8(80 \%)\end{array}$ & $\begin{array}{c}30 \\
\text { hrs(mean } \\
\text { value) }\end{array}$ & $\begin{array}{c}\text { 18hrs(mean } \\
\text { val) }\end{array}$ & $2(20 \%)$ & nill \\
\hline
\end{tabular}

Gr-A(n=10) Del Nido Cardioplegia

\begin{tabular}{|c|c|c|c|c|c|c|c|c|c|}
\hline $\begin{array}{l}\text { Sl. } \\
\text { no }\end{array}$ & $\begin{array}{l}\text { Age } \\
\text { (year) }\end{array}$ & $\begin{array}{c}\text { Sex } \\
\text { (Male } \\
\text { /Female) }\end{array}$ & Case type & $\begin{array}{l}\text { Bypass time } \\
\text { (minutes) }\end{array}$ & $\begin{array}{l}\text { X-clamp time } \\
\text { (minutes) }\end{array}$ & $\begin{array}{l}\text { Events } \\
\text { VT/VF }\end{array}$ & $\begin{array}{l}\text { Inotropic } \\
\text { support }\end{array}$ & $\begin{array}{c}\text { Ventilatory } \\
\text { support (hrs) }\end{array}$ & $\begin{array}{c}\text { Outcomes } \\
\text { (Recovery/de } \\
\text { ath) }\end{array}$ \\
\hline 1 & 28 & $\mathrm{M}$ & $\begin{array}{l}\text { Aortic stenosis with } \\
\text { regurgitation }\end{array}$ & 84 & 64 & $\begin{array}{l}\text { Normal } \\
\text { Cardiac } \\
\text { function }\end{array}$ & High & 42 & $\begin{array}{c}\text { Smooth } \\
\text { Recovery }\end{array}$ \\
\hline 2 & 37 & $\mathrm{M}$ & $\begin{array}{l}\text { Severe aortic stenosis, with } \\
\text { LVH(LVIDD }=60, \text { LVIDS }= \\
42)\end{array}$ & 88 & 69 & $\begin{array}{c}\text { VT \& VF } \\
\text { with } \\
\text { Low CO, } \\
\text { Cardiogeni } \\
\text { c shock }\end{array}$ & High & 18 & $\begin{array}{l}\text { Death on } 2 \text { nd } \\
\text { post op day }\end{array}$ \\
\hline 3 & 36 & $\mathrm{~F}$ & $\begin{array}{c}\text { Severe aortic stenosis with } \\
\text { calcified }\end{array}$ & 68 & 58 & $\begin{array}{l}\text { Normal } \\
\text { Cardiac } \\
\text { function }\end{array}$ & Moderate & 39 & Recovery \\
\hline 4 & 48 & $\mathrm{M}$ & $\begin{array}{l}\text { Severe aortic stenosis with } \\
\text { regurgitation }\end{array}$ & 90 & 70 & $\begin{array}{l}\text { Normal } \\
\text { Cardiac } \\
\text { function }\end{array}$ & Moderate & 18 & Recovery \\
\hline 5 & 52 & $\mathrm{M}$ & Severe aortic stenosis & 91 & 64 & $\begin{array}{l}\text { Normal } \\
\text { Cardiac } \\
\text { function }\end{array}$ & High & 28 & Recovery \\
\hline 6 & 35 & $\bar{M}$ & $\begin{array}{c}\text { Severe aortic stenosis with } \\
\text { concentric LVH }\end{array}$ & 82 & 55 & $\mathrm{VT} / \mathrm{VF}$ & Moderate & 38 & Recovery \\
\hline 7 & 34 & $\mathrm{M}$ & $\begin{array}{c}\text { Severe aortic stenosis with } \\
\text { calcified }\end{array}$ & 98 & 70 & $\begin{array}{l}\text { Normal } \\
\text { Cardiac } \\
\text { function }\end{array}$ & High & 24 & Recovery \\
\hline 8 & 32 & $\mathrm{~F}$ & $\begin{array}{l}\text { Severe aortic stenosis with } \\
\text { concentric LVH }\end{array}$ & 110 & 69 & $\mathrm{VT} / \mathrm{VF}$ & High & 12 & Recovery \\
\hline 9 & 37 & $\mathrm{M}$ & $\begin{array}{c}\text { Severe aortic stenosis, with } \\
\text { calcified }\end{array}$ & 94 & 64 & $\begin{array}{l}\text { Normal } \\
\text { Cardiac } \\
\text { function }\end{array}$ & Moderate & 33 & Recovery \\
\hline 10 & 34 & $\mathrm{M}$ & $\begin{array}{l}\text { Severe aortic stenosis with } \\
\text { concentric LVH }\end{array}$ & 95 & 67 & VT/VF & High & $\begin{array}{c}14 \text { hrs then } \\
\text { reintubation } 34 \\
\text { hrs }\end{array}$ & $\begin{array}{l}\text { Death on 3rd } \\
\text { post op day }\end{array}$ \\
\hline
\end{tabular}


Gr-B HTK Cardioplegia( $\mathrm{n}=10)$

\begin{tabular}{|c|c|c|c|c|c|c|c|c|c|}
\hline Sl.no & Age(years) & $\begin{array}{c}\text { Sex } \\
\text { (Male/F } \\
\text { emale) }\end{array}$ & Case type & $\begin{array}{l}\text { Bypass time } \\
\text { (minutes) }\end{array}$ & $\begin{array}{l}\text { X-clamp } \\
\text { time } \\
\text { (minutes) }\end{array}$ & $\begin{array}{l}\text { Events } \\
\text { VT/VF }\end{array}$ & $\begin{array}{l}\text { Inotropic } \\
\text { support }\end{array}$ & $\begin{array}{c}\text { Ventilatory } \\
\text { support(Hrs) }\end{array}$ & Outcomes \\
\hline 1 & 29 & $\mathrm{~F}$ & $\begin{array}{c}\text { Severe aortic stenosis with } \\
\text { calcified }\end{array}$ & 74 & 54 & Nil & Moderate & 20 & Smooth recovery \\
\hline 2 & 44 & M & Severe AS,AR & 78 & 50 & Nil & Minimum & 17 & Smooth recovery \\
\hline 3 & 39 & $\mathrm{M}$ & Severe AS with LVH & 78 & 55 & Nil & Minimum & 18 & Smooth recovery \\
\hline 4 & 27 & $\mathrm{M}$ & $\begin{array}{c}\text { Severe aortic stenosis with } \\
\text { calcified }\end{array}$ & 87 & 64 & Nil & Minimum & 21 & Recovery \\
\hline 5 & 32 & $\mathrm{~F}$ & $\begin{array}{l}\text { Severe AS,AR with } \\
\text { calcified }\end{array}$ & 80 & 56 & Nil & Minimum & 14 & Smooth recovery \\
\hline 6 & 46 & $\mathrm{~F}$ & Severe AS with LVH & 82 & 62 & Nil & Moderate & 17 & Recovery \\
\hline 7 & 38 & $\mathrm{M}$ & Severe AS & 93 & 62 & Nil & Minimum & 22 & Recovery \\
\hline 8 & 41 & $\mathrm{~F}$ & Calcified AS & 90 & 58 & Nil & Minimum & 18 & Smooth recovery \\
\hline 9 & 33 & M & $\begin{array}{c}\text { Severe AS,AR with } \\
\text { calcified }\end{array}$ & 94 & 67 & Nil & Minimum & 24 & Recovery \\
\hline 10 & 43 & $\mathrm{M}$ & Calcified AS & 108 & 68 & Nil & Minimum & 13 & Recovery \\
\hline
\end{tabular}

\section{Discussion}

Cardioplegia administration and proper myocardial protection is a challenging job in the sevre aortic stenosis associated with ventricular hypertrophy. Left ventricular chamber stiffness increases during pressure overload hypertrophy and this enhances wall thickness.

Several studies show that cardiac hypertrophy is an risk factor for the development of sudden death, myocardial infarction and heart failure ${ }^{(8)}$ Moreover, the cardiac hypertrophy responsible for creating an arrhythmogenic substrate ${ }^{(9)}$ This is also important that hypertrophy alters vulnerability to ischaemia and reperfusion injury ${ }^{(10)}$

These risk factors can be minimised by proper myocardial protection. There are so many myocardial protection strategies and cardioplegia solutions have been using but till now it is not clear which cardioplegia is ideal to protect these type of myocardium. So in our study we have tried to find out by comparing between del Nido and HTK cardioplegia.

In our study, in first 10 consecutive cases, groupA $(n=10)$, we used Del Nido cardioplegia but result was not satisfactory (Table-3). Then we sought the alternative and in another 10 consecutive cases, group-B $(n=10)$, we used HTK cardioplegia and the result was too much satisfactory, $100 \%$ patients were smooth recovered (Table-3).

In our study all surgeries of both group were performed by using cardiopulmonary bypass with moderate systemic hypothermia at 28 to $32^{\circ} \mathrm{C}$.
(11-13). After systemic cooling ' $X$ '-clamp applied on the aorta and myocardial protection was achieved by del Nido/HTK cardioplegia. Topical hypothermia was maintained by cold ice saline . Valves were replaced by St. Jude Mechanical Master series. Average bypass time was 90 minutes and ' $\mathrm{X}$ '-clamp time was 60 minutes.

\section{Conclusion}

Severe aortic stenosis patient who underwent aortic valve replacement under cardiopulmonary bypass using delnido or HTK cardioplegia, the myocardial protection was better maintained using HTK cardioplegia than del Nido cardioplegia in respect of cardiac function, ventilatory support, morbidity and mortality. So we are hopeful that our study will help in the progression of myocardial protection strategy during aortic valve surgery.

Conflict of interest- none declared.

\section{References}

1. Yerebakan et al, Journal of cardiothoracic surgery 2014, 9:141 DOI; 10.1186/s 13019-014-0141-5

2. Modification of ischemia-ReperfusionInduced Injury by Cardioprotective Intervention,18Ming Zhang, MD,Tamer Sallam, BS, BA, Yan-Jun $\mathrm{Xu}, \mathrm{PhD}$, and Naranjan S.Dhalla, PhD,MD (Hon),DSc (Hon). 
3. Patent 5,407,793. Alexandria, PA: U.S. Patent and Trademark Office; 1995.

4. Charette K, Gerrah R, Quaegebeur J, et al. Single dose myocardial protection utilizing del Nido cardioplegia solution during congenital heart surgery procedures. Perfusion. 2012;27:98-103.

5. Matte GS, Del Nido PJ. History and use of del Nido cardioplegia solution at Boston Children's Hospital. J Extra Corpor Technol. 2012;44:98-103.

6. Heinemeyer D, Belles G, Stapenhorst K: Intracellular $\mathrm{pH}$ measurement during cardiac arrest in ventricular myocardium by Bretschneider's cardioplegic solution HTK and St. Thomas Hospital solution with and without procaine. Thorac Cardiovasc Surg 35: 48-52, 1987.

7. Keeling IM, Obermayr RP, Schneider B, Spieckermann PG: Postischemic cardiac function recovery in the isolated rat heart: Effects of adenosine deaminase and nucleoside transport inhibition. Langenbecks Arch Surg 385: 531-537, 2000.

8. Frohlich ED, Apstein C, Chobanian AV, et al. The heart in hypertension. N Engl J Med. 1992 Oct 1;327(14):998-1008.

9. Kozhevnikov D, Caref EB, El-Sherif N. Mechanisms of enhanced arrhythmogenicity of regional ischaemia in the hypertrophied heart. Heart Rhythm. 2009 Apr;6(4):522-527.

10. Sambandam N, Lopaschuk GD, Brownsey RW, Allard MF. Energy metabolism in the hypertrophied heart. Heart Fail Rev. 2002 Apr;7(2):161-173.

11. Gravlee GP, Davis RF, Stammers AH, Ungerleider RM, eds. Cardiopulmonary Bypass: Principles and Practice, 3rd ed Philadelphia, PA: Lippincott Williams \& Wilkins; 2008:172-179, 702-4.

12. Hensley FA, Martin DE, Gravlee GP. A Practical Approach to Cardiac Anesthesia, 5th ed. Philadelphia, PA: Lippincott Williams \& Wilkins; 2013:649-650.

13. Chambers DJ. Mechanisms and alternative methods of achieving cardiac arrest. Ann Thorac Surg.2003;75:S661-S666.

14. Use of del Nido Cardioplegia for Adult Cardiac Surgery at the Cleveland Clinic: Perfusion Implications Kuna Kim, BS, * Clifford Ball, BS,* Patrick Grady, $\mathrm{BS}, *$ and Stephanie Mick, MD $\dagger$ 